
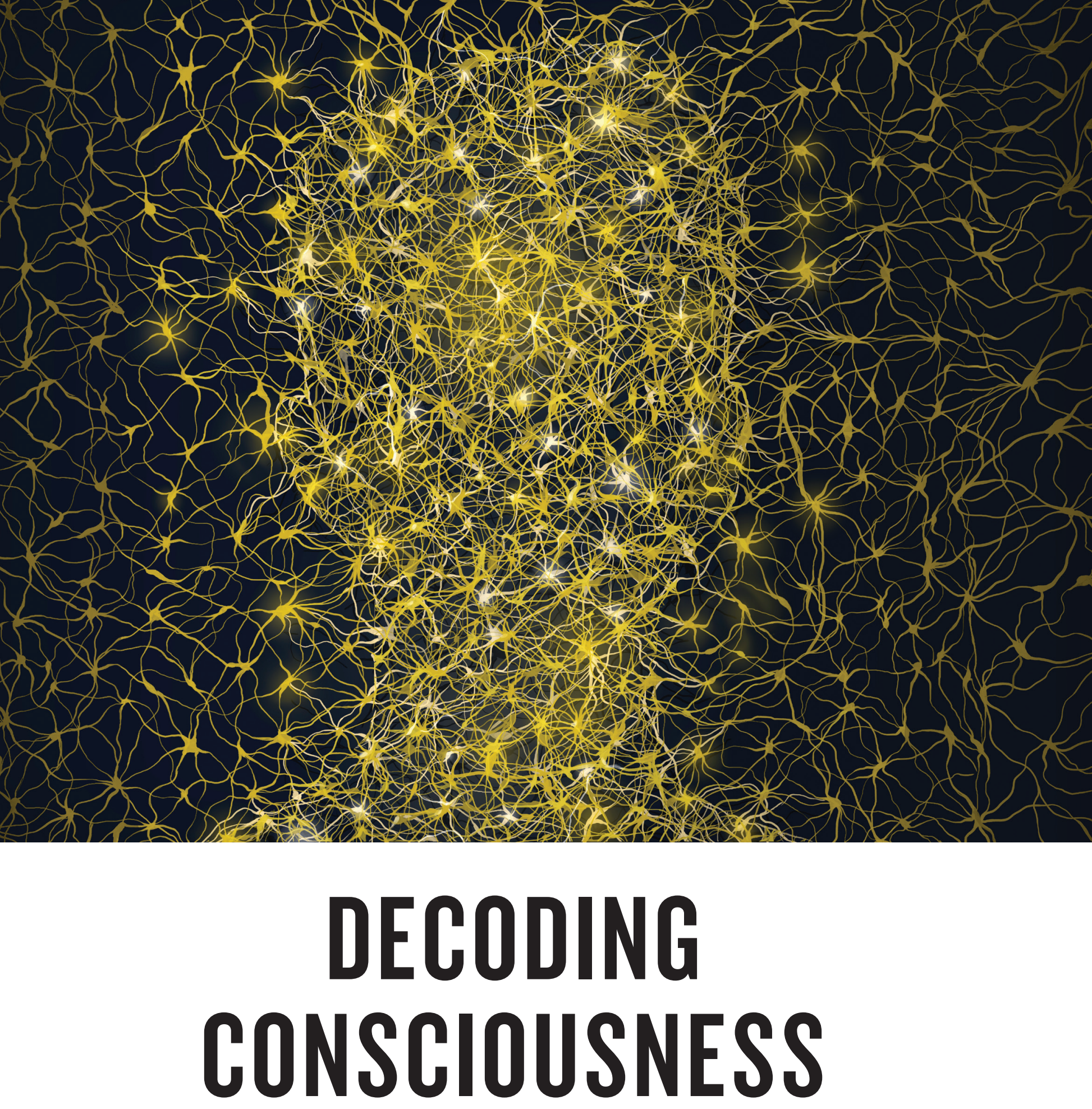

A growing understanding of how consciousness works could lead to fresh treatments for brain injuries and phobias, as well as a deeper understanding of ourselves.

\author{
BY EMILY SOHN
}

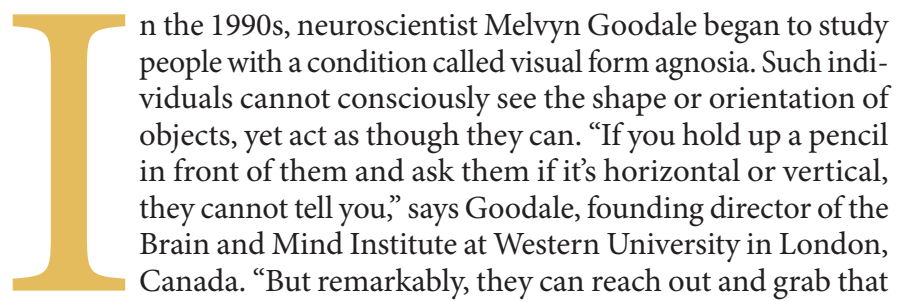


pencil, orienting their hand correctly as they reach out to make contact with it."

Goodale's initial interest related to how the brain processes vision. But as his work to document the two visual systems that govern conscious and unconscious sight progressed, it caught the eye of philosophers, who drew him into conversations about consciousness - a melding of fields that has transformed both.

Newly developed techniques for measuring brain activity are enabling scientists to refine their theories about what consciousness is, how it forms in the brain and where the boundaries lie between being conscious and unconscious. And as our understanding of consciousness improves, some researchers are beginning to build strategies for its manipulation, with the possibility of treating brain injuries, phobias and mental-health conditions such as post-traumatic stress disorder (PTSD) and schizophrenia.

But even as research progresses, and ideas from science and philosophy continue to meld, essential questions remain unanswered. "It's still just fundamentally mysterious how consciousness happens," says Anil Seth, a cognitive and computational neuroscientist and co-director of the Sackler Centre for Consciousness Science at the University of

Sussex in Brighton, UK.

\section{DETECTIVE STORY}

Consciousness is often described as the mind's subjective experience. Whereas a basic robot can unconsciously detect conditions such as col-

our, temperature or sound, consciousness describes the qualitative feeling that is associated with those perceptions, together with the deeper processes of reflection, communication and thought, says Matthias Michel, a philosopher of science and a PhD student at Sorbonne University in Paris.

By the second half of the nineteenth century, scientists had developed a programme for studying consciousness that resembles present approaches, Michel says. But research lulled throughout much of the twentieth century as psychologists rejected introspection to focus instead on observable behaviours and the stimuli that caused them. Even in the 1970s and 1980s, as cognitive science became established, consciousness remained a controversial topic among scientists, who openly questioned whether it was a valid area of scientific investigation. Early in his career, molecular biologist and Nobel laureate Francis Crick wanted to study consciousness, but instead chose to work on the more tangible mysteries of DNA.

Eventually, prominent scientists (including Crick) did decide to tackle consciousness, which ushered in a shift in thinking that surged in the 1990s, fuelled by the increasing availability of brain-scanning technologies such as functional magnetic resonance imaging
(fMRI) and electroencephalography (EEG). At this point, scientists finally embarked on a major search for the mechanisms in the brain that are associated with the conscious processing of information.

A succession of breakthroughs followed, including the case of a 23-year-old woman who sustained a severe brain injury in a car accident in July 2005, which left her in a non-responsive state, also known as wakeful unawareness. She could open her eyes and exhibited cycles of sleep and wakefulness, but did not respond to commands or show signs of voluntary movement. She was still unresponsive five months later. In a first-of-its-kind study, Adrian Owen, a neuroscientist then at the University of Cambridge, UK, and now at Western University, and his colleagues observed the woman using fMRI while giving her a series of verbal commands ${ }^{1}$. When the team asked her to imagine playing tennis, they observed activity in a part of her brain called the supplementary motor area. When they asked her to imagine walking through her home, activity ramped up instead in three areas of the brain that are associated with movement and memory. The researchers observed the same patterns in healthy volunteers who were given identical instructions.

create electrical 'echoes' in the brain that can be recorded using EEG. The technique is similar to knocking on the brain, in the same way that a person might knock on a wall to gauge its thickness, says Martin Monti, a neuroscientist at the University of California, Los Angeles. While a person is under general anaesthesia, or in a dreamless sleep, the echoes that are produced are simple. But in the conscious brain, the echoes are complex and spread widely over the surface of the cerebral cortex (the outer layer of the brain). The work could eventually lead to a tool that is capable of detecting consciousness even in people who can't see, hear or respond to verbal commands.

\section{LOCATION, LOCATION, LOCATION}

As scientists have become more adept at detecting consciousness, they have begun to determine which brain regions and circuits are most important. But there is still much debate about what constitutes consciousness in neural terms, with particular disagreement over which brain processes and regions matter most.

Since at least the nineteenth century, scientists have known that the cerebral cortex is important for consciousness. Fresh evidence has highlighted a posterior-cortical 'hot zone' that is responsible for sensory experiences. For example, in a 2017 sleep study, researchers roused people throughout the night while monitoring them with $\mathrm{EEG}^{4}$. Around $30 \%$ of the time, participants who were jolted from

The finding that some people in a coma show signs of consciousness was transformative for neuroscience, says Seth. The work suggested that some people could understand speech and possibly communicate, even when they seemed not to respond to doctors and family members.

In the years since Owen's study was published, investigations of people with brain injuries have offered more evidence that consciousness is detectable in as many as $10-20 \%$ of people who are non-responsive. In 2010, a study used fMRI to monitor the brains of 54 people in Belgium and the United Kingdom with severe brain injuries ${ }^{2}$. Five showed signs of brain responsiveness when they were instructed to imagine playing tennis or walking through their house or city, a protocol similar to that established by Owen's team five years earlier. Two of those five people did not demonstrate any awareness in conventional bedside assessments.

Scientists have also started to test ways of detecting consciousness without the need to give people verbal instructions. In a series of studies that began in 2013 (ref. 3), neuroscientist Marcello Massimini at the University of Milan and his colleagues have used transcranial magnetic stimulation (TMS) to sleep reported not experiencing anything just before they woke up. The study showed that those people without conscious experiences during sleep had lots of low-frequency activity in the posterior-cortical region of their brains before waking. People who reported that they had been dreaming, however, had less lowfrequency activity and more high-frequency activity. As a result, the researchers suggest that by observing a person's posterior-cortical hot zone during sleep, it might be possible to predict whether they are dreaming - and even the specific contents of their dreams, including faces, speech and movement.

It has become increasingly clear, however, that consciousness is not confined to only one region of the brain. Various cells and pathways are engaged, depending on what is being perceived or the type of perception that is involved. Investigating the coordination of neural signalling might help researchers to find reliable signatures of consciousness. In a 2019 study that collected fMRI data from 159 people, researchers found that, compared with people in minimally conscious states and those under anaesthesia, the brains of healthy individuals had more complex patterns of coordinated signalling that also changed constantly ${ }^{5}$.

Plenty of unknowns remain. Scientists 
disagree about how study results should be interpreted, and measuring whether a person is 'in' or 'out' of consciousness is a challenge that differs from looking at what happens in the brain as it becomes aware of different types of information. Nevertheless, studies of brain function at various levels of consciousness are starting to offer alternative ways of looking at the brain at a mechanistic level. The hope, says Seth, is that consciousness researchers can "move to a more twenty-first century sort of psychiatry, where we can intervene more specifically in the mechanisms to resolve specific symptoms".

\section{TINKERING AND TREATING}

Attempts at intervention are in progress, and people with brain injuries could be among the first to benefit. On the basis of research that points to the thalamus as playing an important part in consciousness, for example, Monti and his colleagues have been experimenting with a non-invasive technique that uses ultrasound to stimulate that region of the brain in people with brain damage.

They conducted their initial test of the procedure on a 25 -year-old man who was in a coma after a car accident 19 days earlier. Within 3 days, the man recovered his ability to understand language, to respond to commands and to answer yes-no questions with head gestures. Five days later, he was trying to walk.

The case report ${ }^{6}$, published in 2016, makes it clear that his recovery could have been a coincidence - people often emerge from comas spontaneously. But unpublished follow-up work suggests that the ultrasound approach probably does make a difference. Monti's team has since performed the thalamus-stimulating procedure on a man with a brain injury who had been involved a car accident several years before. The patient had long been in a minimally conscious state, in which people show some evidence of awareness of their environment or themselves. Several days after the experimental treatment, the man's wife asked him whether he recognized specific people in family photographs. He was able to reliably answer yes by looking up, and no by looking down. Monti remembers visiting the patient and his wife soon after the procedure. "She looked at me and she didn't even say hello. She said, 'I want more'," Monti says. It was the first time that she'd had a conversation with her husband since the accident.

Monti and his colleagues have found similarly encouraging results in several other people in persistent comas, but it is unclear whether the benefits last for more than a few weeks before recipients revert to their original state. The team's work is ongoing, and the researchers are now trying to work out whether repeat treatments will make the benefits last longer. "I really think this is going to prove to be a possible way in which we can help patients recover," Monti says. "Somebody once called it jump-starting the brain. We're

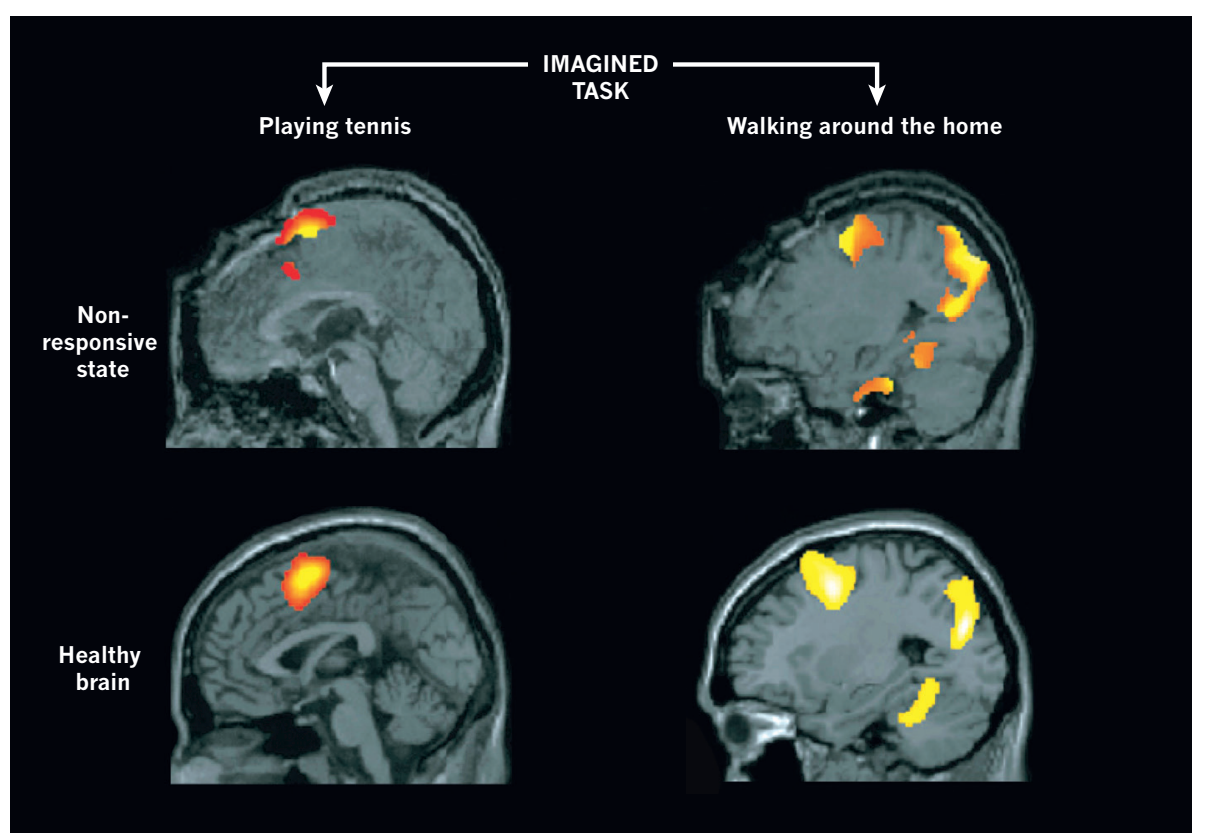

Brain activity in people in an apparently non-responsive state can be similar to that of healthy people.

not quite jump-starting it, but the metaphor is valid."

Further inroads into the mechanisms of consciousness might lead to better treatments for anxiety, phobias and PTSD, suggests work by Hakwan Lau, a neuroscientist at the University of California, Los Angeles, and his colleagues. The standard approach to treating fears is exposure therapy, which pushes people to repeatedly face the thing that scares them the most. But such treatment is unpleasant, and drop-out rates can reach $50 \%$ or more.

Instead, Lau's team is attempting to reprogram the unconscious using an fMRIbased technique that rewards people for

creatures. After the experiment, the sweatiness of people's palms - a trait that reflects their stress levels - in response to seeing these animals, was decreased. Activation of the amygdala, an area of the brain that responds to threats, was also reduced. The technique seemed to have reprogrammed the brain's fear responses outside the conscious awareness of participants.

Lau and his colleagues are testing the procedure on people with phobias, and eventually they hope to use it to treat PTSD. But the technique has a considerable limitation. Despite diminishing physical symptoms, it does not seem to affect how people feel about

\section{"WE'PRENOT QUTEEJUMPPSTARTINGGTHE BRAIN, BUT THEMETAPHOR ISVALID,"}

activating specific brain regions. In a doubleblind trial, the researchers challenged 17 people to make a dot on a computer screen bigger, using any mental strategy ${ }^{7}$. The larger they could make it, the more money they would be paid for completing the study. Participants could think about whatever they wanted. What they didn't know, however, was that the dot would expand only when they activated parts of their brain that, according to previous observations made in a larger group of people, would became active when they saw pictures of animals of which they were afraid, such as spiders or snakes.

Over time, participants became better at activating the correct parts of their brain, but without knowingly thinking of fear-evoking spiders and snakes. "If you ask the patients if they are actually afraid," Lau says, "they say yes."

Ultimately, tackling fear might require targeting both unconscious and conscious pathways, which work in different ways in the brain, says Joseph LeDoux, a neuroscientist at New York University in New York City. The unconscious pathway, he says, emerges from the amygdala. But those hardwired reactions to threats, he suggests, should not be considered as fear at all. Instead, the conscious experience of fear comes from cognitive awareness and the emotional interpretation of a situation. The resulting experiences are not centred on the amygdala. LeDoux says that the difference is evident in people who have blindsight, who cannot consciously perceive visual stimuli but 
act as though they can. When presented with a threat, they exhibit activity in the amygdala together with physical responses. But they don't report feeling afraid.

That disconnect might also offer insight into why current medications for anxiety do not always work as well as people hope, LeDoux says. Developed through animal studies, these medications might target circuits in the amygdala and affect a person's behaviours, such as their level of timidity - making it easier for them to go to social events. But such drugs don't necessarily affect the conscious experience of fear, which suggests that future treatments might need to address both unconscious and conscious processes separately. "We can take a brain-based approach that sees these different kinds of symptoms as products of different circuits, and design therapies that target the different circuits systematically," he says. "Turning down the volume doesn't change the song - only its level."

Psychiatric disorders are another area of interest for consciousness researchers, Lau says, on the basis that some mental-health conditions, including schizophrenia, obsessive-compulsive disorder and depression, might be caused by problems at the unconscious level - or even by conflicts between conscious and unconscious pathways. The link is only hypothetical so far, but Seth has been probing the neural basis of hallucinations with a 'hallucination machine' - a virtual-reality program that uses machine learning to simulate visual hallucinatory experiences in people with healthy brains. Through experiments, he and his colleagues have shown that these hallucinations resemble the types of visions that people experience while taking psychedelic drugs, which have increasingly been used as a tool to investigate the neural underpinnings of consciousness.

If researchers can uncover the mechanisms behind hallucinations, they might be able to manipulate the relevant areas of the brain and, in turn, treat the underlying cause of psychosis - rather than just address the symptoms. By demonstrating how easy it is to manipulate people's perceptions, Seth adds, the work suggests that our sense of reality is just another facet of how we experience the world.

\section{IN SEARCH OF LEGITIMACY}

Every year, tens of thousands of people in the United States become conscious while under general anaesthesia. They cannot move or speak, but they might be able to hear voices or equipment noises, and to feel pain. The experience can be traumatic and is fraught with ethical and legal ramifications for the doctors who are caring for them. Some scientists are working to promote guidelines for communicating with unresponsive patients, as well as ways of looking for signs of discomfort in such people. And they are urging the development of improved training and laws to deal with the possibility that alternative ways of detecting

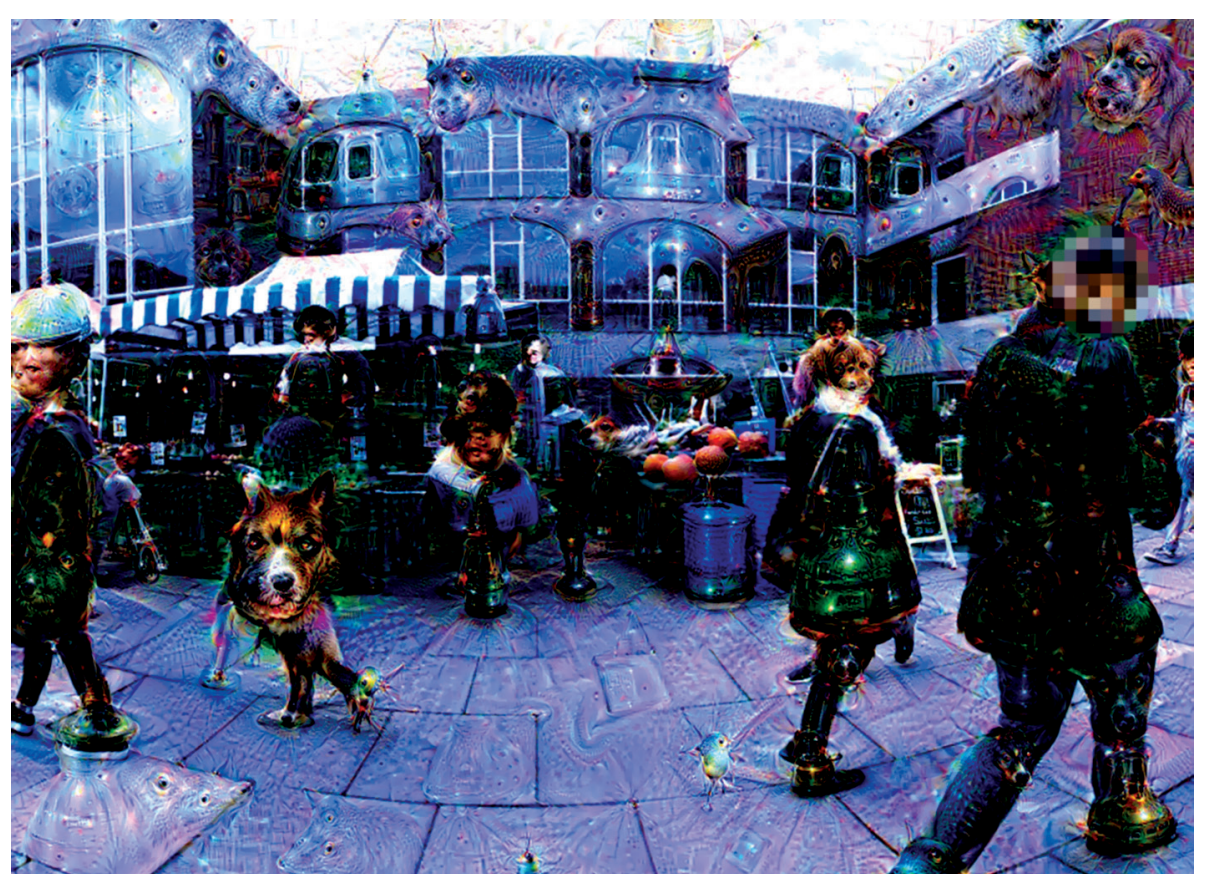

A hallucination created by a machine-learning algorithm that simulates altered visual perception.

consciousness will alter the definition of informed consent for medical procedures.

Researchers are also starting to push for better communication with the public about what consciousness science can and cannot achieve. Michel says that claims that are unsupported by empirical data have proliferated in consciousness research. One, in particular, called integrated information theory, has received plenty of private funding and media attention, even though it has been dismissed by him and other experts in the field. In an informal survey of 249 researchers in 2018, Michel and his colleagues found that around $22 \%$ of those who had not published papers or attended major meetings on consciousness - and were therefore deemed to be non-experts - trusted integrated information theory ${ }^{8}$. Michel suspects that a 'guru effect' could be to blame, with non-experts thinking that complex and obscure statements made by intelligent people who project authority are more likely to be true than simpler ideas. "In a sense, the apparent complexity of the theory is used as a proxy for its probability of being true," Michel says. "They don't really understand it, but they come to believe that if they understood it, they would likely consider it as the right theory of consciousness."

To solidify the legitimacy of consciousness science and to encourage acceptance of evidence-based ideas, he and a group of $57 \mathrm{col}-$ leagues from a range of disciplines - including Seth, Lau, Goodale and LeDoux — followed up the informal study with a 2019 paper that reviewed the state of the field ${ }^{9}$. Its findings were mixed. Consciousness research is not yet recognized as a strategically focused area by the US National Institute of Mental Health, they wrote. Job creation in the field has lagged behind other nascent disciplines such as neuroeconomics and social neuroscience. And public funding, especially in the United States, has been relatively scarce. But certain areas are gaining attention. Since the mid-2000s, the US National Institutes of Health has provided several large grants to support research that addresses, among other important topics, the neurological differences between consciousness and being in a coma, or wakefulness and being asleep. Such studies might offer a window on the neural signatures of consciousness. Some major private philanthropic foundations and organizations are also supporting research on big ideas in consciousness, says Goodale, who receives funding from one such charitable organization, the Canadian Institute for Advanced Research in Toronto.

As funding and publications accumulate, scientists have become increasingly able to make investigations into consciousness a reasonable - if not central - part of their research plan, Seth says. "There has been a general assimilation of consciousness within the standard practice of neuroscience and psychology and medicine," he says. "It has become more normalized, which is a good thing."

Emily Sohn is a freelance journalist in Minneapolis, Minnesota.

1. Owen, A. M. et al. Science 313, 1402 (2006).

2. Monti, M. M. et al. N. Engl. J. Med. 362, 579-589 (2010).

3. Casali, A. G. et al. Sci. Transl. Med. 5, 198ra105 (2013).

4. Siclari, F. et al. Nature Neurosci. 20, 872-878 (2017).

5. Demertzi, A. et al. Sci. Adv. 5, eaat7603 (2019).

6. Monti, M. M., Schnakers, C., Korb, A. S., Bystritsky, A \& Vespa, P. M. Brain Stimul. 9, 940-941 (2016).

7. Taschereau-Dumouchel, V. et al. Proc. Natl Acad. Sci. USA 115, 3470-3475 (2018).

8. Michel, M. et al. Front. Psychol. 9, 2134 (2018).

9. Michel, M. et al. Nature Hum. Behav. 3, 104-107 (2019). 